\title{
On the sectional curvature of lightlike submanifolds
}

\author{
Erol Kılıç and Mehmet Gülbahar ${ }^{2 *}$
}

\author{
"Correspondence: \\ mehmetgulbahar85@gmail.com \\ ${ }^{2}$ Department of Mathematics, \\ Faculty of Arts and Sciences, Siirt \\ University, Siirt, 56100, Turkey \\ Full list of author information is \\ available at the end of the article
}

\begin{abstract}
The main purpose of this paper is to show how to obtain rigidity theorems with the help of curvature invariants in submanifolds of a semi-Riemannian manifold. For this purpose, the bounded sectional curvature is introduced and some special submanifolds of $r$-lightlike submanifolds of a semi-Riemannian manifold are investigated.
\end{abstract}

MSC: 53C40; 53C42; 53C50

Keywords: curvature; lightlike submanifold; semi-Riemannian manifold

\section{Introduction}

In 1979, Kulkarni [1] proved that the sectional curvature of a semi-Riemannian manifold $M$ is unbounded from above and below at each point unless the manifold has constant sectional curvature. Later, Nomizu [2] showed that if there exists a real number $d$ such that at any point $p \in M$, the sectional curvature $K(\Pi)$ of a 2-plane section $\Pi$ satisfies

$$
K(\Pi) \leq d,
$$

then $M$ is of constant sectional curvature.

In [3], Dajczer and Nomizu, and in [4], Harris remarked that if the absolute value of the sectional curvature $|K(\Pi)|$ is bounded for all timelike 2-planes $\Pi$ (or for all spacelike 2-planes $\Pi$ ) at $p \in M$, then $M$ is of constant sectional curvature.

In Riemannian geometry, there are various relations between the intrinsic and extrinsic curvature invariants of a submanifold, known as Chen inequalities, in the literature [544]. But different from the Riemannian context, from the Kulkarni result, it is too restrictive to relate the intrinsic invariant of a submanifold with the extrinsic ones for a submanifold of a semi-Riemannian manifold. This reveals the necessity to re-investigate or modify the domain of sectional curvature map in semi-Riemannian geometry and lightlike geometry. For this purpose, the authors showed in $[45,46]$ that the domain of the sectional curvature map in a Lorentzian manifold is not a linear subspace as it was used in the literature but it is a polynomial subspace of a projective vector space which makes it possible for the sectional curvature map on any Lorentzian manifold to be bounded. This is revolutionary information which might lead one to require a revision for many studies related to the sectional curvature map in semi-Riemannian geometry and lightlike geometry.

(c) 2016 KIlıç and Gülbahar. This article is distributed under the terms of the Creative Commons Attribution 4.0 International License (http://creativecommons.org/licenses/by/4.0/), which permits unrestricted use, distribution, and reproduction in any medium, provided you give appropriate credit to the original author(s) and the source, provide a link to the Creative Commons license, and indicate if changes were made. 
In this paper, we extend this modified sectional curvature from Lorentzian manifolds to semi-Riemannian manifolds under the name of 'bounded sectional curvature'. We introduce some special $r$-lightlike submanifolds and establish some relationships involving intrinsic curvatures and extrinsic curvatures for $r$-lightlike submanifolds of a semiRiemannian manifold.

\section{Preliminaries}

Let $(\tilde{M}, \tilde{g})$ be a real $(\tilde{m}+\tilde{n})$-dimensional semi-Riemannian manifold, where $\tilde{n} \geq 1, \tilde{m}>1$ with $\tilde{g}$ a semi-Riemannian metric on $\tilde{M}$ of constant index $\tilde{q} \in\{1, \ldots, \tilde{m}+\tilde{n}-1\}$. Suppose $(M, g)$ to be an $\tilde{n}$-dimensional lightlike submanifold of $(\tilde{M}, \tilde{g})$ where $g$ denotes the restriction of $\tilde{g}$ to $M$ which we assume be degenerate. Then there exists a smooth distribution, called radical space of the tangent space $T_{p} M$ at $p \in M$, defined by

$$
\operatorname{Rad} T_{p} M=T_{p} M \cap T_{p} M^{\perp} \neq\{0\}
$$

where

$$
T_{p} M^{\perp}=\left\{v_{p} \in T_{p} \tilde{M}: \tilde{g}_{p}\left(v_{p}, w_{p}\right)=0, \forall w_{p} \in T_{p} M\right\} .
$$

Let us consider the rank of $\operatorname{Rad} T_{p} M$ to be $r(r>0), \tilde{n}=n+r(n \geq 0)$, and $\tilde{m}=n+m+2 r$ $(m \geq 0)$. Then there exist the following four possible cases:

Case 1. $M$ is called a $r$-lightlike submanifold if $1 \leq r<\min \{n+r, m+r\}$.

Case 2. $M$ is called a coisotropic submanifold if $m=0$.

Case 3. $M$ is called a isotropic submanifold if $n=0$.

Case 4. $M$ is called a totally lightlike submanifold if $m=n=0$.

Let $(M, g)$ be an $(n+r)$-dimensional lightlike submanifold of $(\tilde{M}, \tilde{g})$. Let $S(T M)$ and $S\left(T M^{\perp}\right)$ be a complementary non-degenerate vector bundle of $\operatorname{Rad} T M$ in $T M$ and $T M^{\perp}$, respectively, $\operatorname{tr}(T M)$ be a complementary vector bundle to $T M$ in $\left.T \tilde{M}\right|_{M}$. Then we have

$$
\left.T \tilde{M}\right|_{M}=(\operatorname{Rad} T M \oplus \operatorname{ltr}(T M)) \oplus_{\mathrm{orth}} S(T M) \oplus_{\mathrm{orth}} S\left(T M^{\perp}\right)
$$

where $\oplus_{\text {orth }}$ denotes the orthogonal direct sum and $\oplus$ denotes the direct sum, but it is not orthogonal.

For any $r$-lightlike submanifold, there exists a local quasi-orthonormal frame field $\left\{\xi_{1}, \ldots, \xi_{r}, e_{1}, \ldots, e_{n}, N_{1}, \ldots, N_{r}, u_{1}, \ldots, u_{m}\right\}$ on a local coordinate neighborhood of $\mathcal{U}$ of $M$ such that this basis satisfies the following relation:

$$
\tilde{g}\left(N_{i}, \xi_{j}\right)=\delta_{i j}, \quad \tilde{g}\left(N_{i}, N_{j}\right)=\tilde{g}\left(N_{i}, u_{j}\right)=\tilde{g}\left(\xi_{i}, u_{j}\right)=0, \quad \forall i, j \in\{1, \ldots, r\}
$$

where $\delta_{i j}$ is the Kronecker delta function and

$$
\begin{array}{ll}
\Gamma\left(\left.\operatorname{Rad} T M\right|_{\mathcal{U}}\right)=\operatorname{Span}\left\{\xi_{1}, \ldots, \xi_{r}\right\}, & \Gamma\left(\left.\operatorname{ltr}(T M)\right|_{\mathcal{U}}\right)=\operatorname{Span}\left\{N_{1}, \ldots, N_{r}\right\} \\
\Gamma\left(\left.S(T M)\right|_{\mathcal{U}}\right)=\operatorname{Span}\left\{e_{1}, \ldots, e_{n}\right\}, & \Gamma\left(\left.S\left(T M^{\perp}\right)\right|_{\mathcal{U}}\right)=\operatorname{Span}\left\{u_{1}, \ldots, u_{m}\right\}
\end{array}
$$


Let $\widetilde{\nabla}$ be the Levi-Civita connection of $\widetilde{M}$ and $P$ be the projection morphism of $\Gamma(T M)$ to $\Gamma(S(T M))$. The Gauss and Weingarten formulas are given by

$$
\begin{aligned}
& \widetilde{\nabla}_{X} Y=\nabla_{X} Y+\sum_{l=1}^{r} B^{l}(X, Y) N_{l}+\sum_{\alpha=1}^{m} D^{\alpha}(X, Y) u_{\alpha} \\
& \widetilde{\nabla}_{X} N_{k}=-A_{N_{k}} X+\sum_{l=1}^{r} \rho_{k l}(X) N_{l}+\sum_{\alpha=1}^{m} \rho_{\alpha k}(X) u_{\alpha} \\
& \widetilde{\nabla}_{X} u_{\beta}=-A_{u_{\beta}} X+\sum_{l=1}^{r} \varepsilon_{\beta l}(X) N_{l}+\sum_{\alpha=1}^{m} \varepsilon_{\beta \alpha}(X) u_{\alpha} \\
& \nabla_{X} P Y=\nabla_{X}^{*} P Y+\sum_{l=1}^{r} C^{l}(X, P Y) \xi_{l} \\
& \nabla_{X} \xi_{k}=-A_{\xi_{k}}^{*} X-\sum_{l=1}^{r} \rho_{k l}(X) \xi_{l}
\end{aligned}
$$

for any $X, Y \in \Gamma(T M)$, where $\nabla$ and $\nabla^{*}$ are the induced linear connection on $T M$ and $S(T M)$, respectively; $B^{l}$ and $D^{\alpha}$ are coefficients of the lightlike second fundamental form and coefficients of the screen second fundamental form of $T M$, respectively, $C^{l}$ are the coefficients of the local second fundamental form on $S(T M), A_{N_{l}}, A_{u_{\alpha}}$ are the shape operators on $M, A_{\xi_{k}}^{*}$ is the shape operator on $S(T M)$ and $\varepsilon_{l}, \varepsilon_{\alpha}, \rho_{l}, \rho_{\alpha}$ are 1-forms on $M$ [47].

The second fundamental form $h$ and the local second fundamental form $h^{*}$ are given by

$$
h(X, Y)=\sum_{l=1}^{r} B^{l}(X, Y) N_{l}+\sum_{\alpha=1}^{m} D^{\alpha}(X, Y) u_{\alpha}
$$

and

$$
h^{*}(X, P Y)=\sum_{l=1}^{r} C^{l}(X, P Y) \xi_{l}
$$

respectively. The submanifold $(M, g, S(T M))$ is called totally geodesic if

$$
h(X, Y)=0
$$

for all $X, Y \in \Gamma(T M)$ and it is called totally umbilical [48] if there exists a smooth transversal vector field $H \in \Gamma(\operatorname{tr}(T M))$ such that

$$
h(X, Y)=\tilde{g}(X, Y) H
$$

for all $X, Y \in \Gamma(T M)$.

Let $\left\{e_{1}, \ldots, e_{n}\right\}$ be an orthonormal basis of $\Gamma(S(T M))$. Consider

$$
\mu_{1}=\frac{1}{n} \sum_{i=1}^{n} \sum_{l=1}^{r} \varepsilon_{i} B^{l}\left(e_{i}, e_{i}\right) \quad \text { and } \quad \mu_{2}=\frac{1}{n} \sum_{i=1}^{n} \sum_{\alpha=1}^{r} \varepsilon_{i} \varepsilon_{\alpha} D^{\alpha}\left(e_{i}, e_{i}\right) \text {, }
$$


where $\varepsilon_{i}=g\left(e_{i}, e_{i}\right), \varepsilon_{\alpha}=g\left(e_{\alpha}, e_{\alpha}\right)$ for any $i \in\{1, \ldots, n\}$ and $\alpha \in\{1, \ldots, m\}$. The mean curvature vectors on $T M$ and on $\Gamma(S(T M))$ at $p \in M$, denoted by $H(p)$ and $H^{*}(p)$, are given by

$$
\begin{aligned}
& H(p)=\left.\frac{1}{n} \operatorname{trace}\right|_{S(T M)} h=\sum_{l=1}^{r} \mu_{1} N_{l}+\sum_{\alpha=1}^{m} \mu_{2} u_{\alpha}, \\
& H^{*}(p)=\frac{1}{n} \sum_{i=1}^{n} \varepsilon_{i} \sum_{\ell=1}^{r} C^{\ell}\left(e_{i}, e_{i}\right) \xi_{\ell}+\frac{1}{n} \sum_{l=1}^{r} \mu_{1} N_{l}+\frac{1}{n} \sum_{\alpha=1}^{m} \mu_{2} u_{\alpha},
\end{aligned}
$$

respectively. From equation (16), we can see that the submanifold is minimal if and only if $H(p)$ vanishes identically and $D^{\alpha}=0$ on $\operatorname{Rad}(T M)[49,50]$.

Let us denote curvature tensors of the ambient manifold and the submanifold by $\widetilde{R}$ and $R$, respectively. Then the following relation between these tensors holds:

$$
\begin{aligned}
\tilde{g}(\widetilde{R}(X, Y) P Z, P W)= & g(R(X, Y) P Z, P W)+\sum_{l=1}^{r} B^{\ell}(X, P Z) C^{\ell}(Y, P W) \\
& -\sum_{l=1}^{r} B^{l}(Y, P Z) C^{l}(X, P W) \\
& +\sum_{\alpha=1}^{m} \varepsilon_{\alpha}\left[D^{\alpha}(X, P Z) D^{\alpha}(Y, P W)-D^{\alpha}(Y, P Z) D^{\alpha}(X, P W)\right]
\end{aligned}
$$

for all $X, Y, Z, U \in \Gamma(T M)[47]$.

Let $\Pi=\operatorname{Span}\{X, Y\}$ be a 2-dimensional non-degenerate plane in $T_{p} M$. Then the sectional curvature at $p$ is expressed by

$$
K(\Pi)=\frac{g\left(R_{p}(X, Y) Y, X\right)}{g_{p}(X, X) g_{p}(Y, Y)-g_{p}(X, Y)^{2}} .
$$

We note that since $C^{l}$ is not symmetric, the sectional curvature map does not need to be symmetric on any lightlike submanifold of a semi-Riemannian manifold [47].

Now, we recall the following result [51].

Theorem 1 Let $(M, g, S(T M))$ be an r-lightlike submanifold of a semi-Riemannian manifold $(\tilde{M}, \tilde{g})$. Then the following assertions are equivalent:

(i) $S(T M)$ is integrable.

(ii) $h^{*}$ is symmetric on $\Gamma(S(T M))$.

(iii) $A_{N}$ is self-adjoint on $\Gamma(S(T M))$ with respect to $g$.

As a consequence of Theorem 1, we obtain the following theorem.

Theorem 2 Let $(M, g, S(T M))$ be an r-lightlike submanifold of a semi-Riemannian manifold $(\widetilde{M}, \tilde{g})$. The sectional curvature map is symmetric if and only if $S(T M)$ is integrable.

\section{Bounded sectional curvature}

We start by taking into consideration a quotient space given by

$$
S(T M) \oplus_{\text {orth }} S(T M) / \operatorname{SL}(2, \mathbb{R}),
$$


where $\mathrm{SL}(2, R)$ denotes the special linear transformation. For any given two vector pairs $(X, Y)$ and $(A, B)$ in this space, $(X, Y) \sim(A, B)$ if $A=a X+b Y$ and $B=c X+d Y$ with $a d-b c=1$. It is clear that the $\sim$ relation is an equivalence relation. Furthermore, for this relation in this space, we can write $(X, Y) \sim(A, B)$ if and only if $A \wedge B=X \wedge Y$, where $\wedge$ is the wedge product. Since $X \wedge Y$ is an element of the vector space of anti-symmetric contravariant two tensors $\wedge^{2} S(T M)$ which is also known as the second exterior power of $S(T M)$ [52], any element of $S(T M) \oplus_{\text {orth }} S(T M) / \mathrm{SL}(2, \mathbb{R})$ can be considered as an element of $\wedge^{2} S(T M)$ satisfying

$$
\Pi \wedge \Pi=0
$$

for all $\Pi \in \wedge^{2} S(T M)$. We note that equation (21) holds because of the property of antisymmetry of the wedge product. Thus, we have

$$
(S(T M) \wedge S(T M)) / \operatorname{SL}(2, \mathbb{R}) \cong\left\{\Pi \in \wedge^{2} S(T M): \Pi \wedge \Pi=0\right\} .
$$

Now, we consider the space of planes in $S(T M)$. It is well known that any vector pair spanned a plane section in $S(T M)$ are related by a general linear group $\mathrm{GL}(2, \mathbb{R})$. Therefore, the space of planes in $S(T M)$, denoted by the Grassmanian $G_{r_{2}}(S(T M)$ ), is given by

$$
G_{r_{2}}(S(T M)) \equiv\left(S(T M) \oplus_{\text {orth }} S(T M)\right) / \mathrm{GL}(2, \mathbb{R}) .
$$

Since the Grassmanian can be embedded into the real projective space $\mathbb{P}\left(\wedge^{2} S(T M)\right)$ but is not embedded into the space $\wedge^{2} S(T M)$ (this embedding is also known as the Plücker embedding [52]) it can be written

$$
G_{r_{2}}(S(T M))=\left\{\Pi=X \wedge Y \in \mathbb{P}\left(\wedge^{2} S(T M)\right): \Pi \wedge \Pi=0\right\} .
$$

Eventually, if $S(T M)$ is semi-Riemannian, then the sectional curvature map is defined by

$$
K: G_{r_{2}}(S(T M)) \cap\{\Pi=X \wedge Y: G(\Pi, \Pi) \neq 0\} \rightarrow \mathbb{R},
$$

where

$$
G(\Pi, \Pi)=g(X, X) g(Y, Y)-g(X, Y)^{2} .
$$

In the case of $S(T M)$ is Riemannian, then $G(\Pi, \Pi) \neq 0$ for all $\Pi \in \mathbb{P}\left(\wedge^{2} S(T M)\right)$ and thereby the sectional curvature map in the Riemannian context is given by

$$
K: G_{r_{2}}(S(T M)) \rightarrow \mathbb{R}
$$

As a consequence of the above information, we give the following definition.

Definition 1 Let $(M, g, S(T M))$ be an $(n+r)$-dimensional $r$-lightlike submanifold of an $\widetilde{m}$-dimensional semi-Riemannian manifold $(\widetilde{M}, \tilde{g})$ and $S(T M)$ be integrable. The map

$$
K: G_{r_{2}}(S(T M)) \cap\{\Pi: G(\Pi, \Pi) \neq 0\} \rightarrow \mathbb{R}
$$


which is defined by

$$
K(\Pi)=\frac{R(\Pi, \Pi)}{G(\Pi, \Pi)}
$$

is called bounded sectional curvature map.

Proposition 1 Let $(M, g)$ be an $(n+r)$-dimensional $r$-lightlike submanifold of an $\tilde{m}$-dimensional semi-Riemannian manifold $(\tilde{M}, \tilde{g})$ and $S(T M)$ be integrable. Then the bounded sectional curvature map is well defined, bounded, and independent of the choice of basis on П.

Proof Let $\left\{e_{a_{1}} \wedge e_{a_{2}}: a_{1}<a_{2}\right\}$ be a basis $\wedge^{2} S(T M)$. Suppose that $\Pi=e_{a_{1}} \wedge e_{a_{2}}=e_{a_{1}^{\prime}} \wedge e_{a_{2}^{\prime}}$. Then one can write

$$
\begin{aligned}
& e_{a_{1}^{\prime}}=a e_{a_{1}}+b e_{a_{2}}, \\
& e_{a_{2}^{\prime}}=c e_{a_{1}}+d e_{a_{2}},
\end{aligned}
$$

with $a d-b c \neq 0$. Here, it is clear that the area obeys

$$
G\left(e_{a_{1}^{\prime}} \wedge e_{a_{2}^{\prime}}, e_{a_{1}^{\prime}} \wedge e_{a_{2}^{\prime}}\right)=(a d-b c)^{2} G\left(e_{a_{1}} \wedge e_{a_{2}}, e_{a_{1}} \wedge e_{a_{2}}\right) .
$$

Since $S(T M)$ is integrable and $R$ is symmetric, we have

$$
R\left(e_{a_{1}^{\prime}}, e_{a_{2}^{\prime}}, e_{a_{2}^{\prime}}, e_{a_{1}^{\prime}}\right)=(a d-b c)^{2} R\left(e_{a_{1}}, e_{a_{2}}, e_{a_{2}}, e_{a_{1}}\right),
$$

which implies that $K(\Pi)$ is independent of the choice of basis on $\Pi$, it is well defined and both bounded from above or bounded from below.

\section{Special lightlike submanifolds}

We begin this section with the following definition of $[53,54]$.

Definition 2 Let $(\widetilde{M}, \bar{g})$ be an $\widetilde{m}$-dimensional semi-Riemannian manifold of index $\tilde{q}$. A distribution on $\widetilde{M}$ is called maximally timelike if it is timelike and has rank $\tilde{q}$. A distribution on $\widetilde{M}$ is called maximally spacelike if it is spacelike and has rank $(\widetilde{m}-\tilde{q})$.

Now, we recall the following theorem and proposition of Baum in [53].

Theorem 3 (Existence of maximally timelike-spacelike distributions) Let $(\tilde{M}, \tilde{g})$ be a semiRiemannian manifold. Then there is a $\tilde{g}$-orthogonal decomposition such that $T \tilde{M}=\tilde{\mathcal{V}} \oplus_{\text {orth }}$ $\widetilde{\mathcal{H}}$, where $\widetilde{\mathcal{V}}$ is a maximally timelike and $\widetilde{\mathcal{H}}$ is a maximally spacelike distribution on $\widetilde{M}$.

Proposition 2 (Maximally timelike-spacelike distributions are isomorphic) Let $(\widetilde{M}, \tilde{g})$ be a semi-Riemannian manifold. Every maximally timelike (or spacelike) distributions on $\widetilde{M}$ are isomorphic as smooth vector bundles over $\widetilde{M}$. 
Let $(M, g, S(T M))$ be an $(n+r)$-dimensional $r$-lightlike submanifold and $S(T M)$ be an integrable distribution of index $q$. Consider $\left\{e_{1}, \ldots, e_{q}, e_{q+1}, \ldots, e_{n}\right\}$ to be an orthonormal basis of $S(T M)$. Then there exists a $g$-orthogonal decomposition given by

$$
S(T M)=\mathcal{V} \oplus_{\text {orth }} \mathcal{H}
$$

where $\mathcal{V}=\operatorname{Span}\left\{e_{1}, \ldots, e_{q}\right\}$ is the maximally timelike distribution and $\mathcal{H}=\operatorname{Span}\left\{e_{q+1}, \ldots, e_{n}\right\}$ is the maximally spacelike distribution.

The aforementioned concepts can be constructed on the coscreen distribution $S\left(T M^{\perp}\right)$. Let $\widetilde{\mathcal{V}}$ be a maximally timelike and $\widetilde{\mathcal{V}}$ be a maximally spacelike distributions on $S\left(T M^{\perp}\right)$. Then there exists also a $\tilde{g}$-orthogonal decomposition of $S\left(T M^{\perp}\right)$ given by

$$
S\left(T M^{\perp}\right)=\widetilde{\mathcal{V}} \oplus_{\text {orth }} \tilde{\mathcal{H}}
$$

where $\tilde{\mathcal{V}}=\operatorname{Span}\left\{\tilde{e}_{1}, \ldots, \tilde{e}_{\tilde{q}}\right\}, \widetilde{\mathcal{H}}=\operatorname{Span}\left\{\tilde{e}_{\tilde{q}+1}, \ldots, \tilde{e}_{m}\right\}$.

From (11), we can write

$$
h(X, Y)=\sum_{l=1}^{r} B^{l}(X, Y) N_{l}+h^{\tilde{\mathcal{V}}}(X, Y)+h^{\tilde{\mathcal{H}}}(X, Y)
$$

where

$$
h^{\widetilde{\mathcal{V}}}(X, Y)=\sum_{\alpha=1}^{\tilde{q}} D^{\alpha}(X, Y) \tilde{e}_{\alpha} \quad \text { and } \quad h^{\widetilde{\mathcal{H}}}(X, Y) \sum_{\alpha=\tilde{q}+1}^{m} D^{\alpha}(X, Y) \tilde{e}_{\alpha}
$$

for all $X, Y \in T M$.

Now, we shall state some special $r$-lightlike submanifolds definitions.

Definition 3 Let $(M, g, S(T M))$ be an $r$-lightlike submanifold of a semi-Riemannian manifold $(\widetilde{M}, \tilde{g})$ of index $(q+\tilde{q})$ and $S(T M)$ be an integrable distribution of index $q$. The submanifold will be called:

1. Timelike $\mathcal{V}$-geodesic if $\left.h^{s}\right|_{\mathcal{V}} ^{\widetilde{\mathcal{V}}_{\times} \mathcal{V}}=0$, i.e., $D^{\alpha}(X, Y)=0$ for all $X, Y \in \mathcal{V}$ and $\alpha \in\{1, \ldots, \tilde{q}\}$.

2. Timelike $\mathcal{H}$-geodesic if $\left.h^{s}\right|_{\mathcal{H} \times \mathcal{H}}=0$, i.e., $D^{\alpha}(X, Y)=0$ for all $X, Y \in \mathcal{H}$ and $\alpha \in\{1, \ldots, \tilde{q}\}$.

3. Timelike mixed geodesic if $\left.h^{s}\right|_{\mathcal{V}} ^{\widetilde{V}_{\times \mathcal{H}}}=0$, i.e., $D^{\alpha}(X, Y)=0$ for all $X \in \mathcal{V}, Y \in \mathcal{H}$ and $\alpha \in\{1, \ldots, \tilde{q}\}$.

4. Timelike geodesic if $h^{\tilde{\mathcal{V}}}=0$, i.e., $D^{\alpha}(X, Y)=0$ for all $X, Y \in T M$ and $\alpha \in\{1, \ldots, \tilde{q}\}$.

5. Timelike screen geodesic if $h^{*} \mid \mathcal{V}=0$, i.e., $C^{l}(X, Y)=0$ for all $X, Y \in \mathcal{V}$ and $l \in\{1, \ldots, r\}$.

6. Spacelike $\mathcal{V}$-geodesic if $\left.h^{s}\right|_{\mathcal{V}} ^{\widetilde{\mathcal{H}}} \mathrm{\mathcal {V }}=0$, i.e., $D^{\alpha}(X, Y)=0$ for all $X, Y \in \mathcal{V}$ and $\alpha \in\{\tilde{q}+1, \ldots, m\}$.

7. Spacelike $\mathcal{H}$-geodesic if $\left.h^{s}\right|_{\mathcal{H} \times \mathcal{H}} ^{\tilde{\mathcal{H}}}=0$, i.e., $D^{\alpha}(X, Y)=0$ for all $X, Y \in \mathcal{H}$ and $\alpha \in\{\tilde{q}+1, \ldots, m\}$.

8. Spacelike mixed geodesic if $\left.h^{s}\right|_{\mathcal{V}} ^{\widetilde{\mathcal{H}}}=0$, i.e., $D^{\alpha}(X, Y)=0$ for all $X \in \mathcal{V}, Y \in \mathcal{H}$, and $\alpha \in\{\tilde{q}+1, \ldots, m\}$. 
9. Spacelike geodesic if $h^{\widetilde{\mathcal{H}}}=0$, i.e., $D^{\alpha}(X, Y)=0$ for all $X, Y \in T M$ and $\alpha \in\{\tilde{q}+1, \ldots, m\}$.

10. Spacelike screen geodesic if $\left.h^{*}\right|_{\mathcal{H}}=0$, i.e., $C^{l}(X, Y)=0$ for all $X, Y \in \mathcal{H}$ and $l \in\{1, \ldots, r\}$.

11. Mixed geodesic if $h^{s} \mid \mathcal{V} \times \mathcal{H}=0$, i.e., $D^{\alpha}(X, Y)=0$ for all $X \in \mathcal{V}, Y \in \mathcal{H}$ and $\alpha \in\{1, \ldots, m\}$.

12. Mixed screen geodesic if $\left.h^{*}\right|_{\mathcal{V} \times \mathcal{H}}=0$, i.e., $C^{l}(X, Y)=0$ for all $X, Y \in T M$ and $l \in\{1, \ldots, r\}$.

We also note that the submanifold is:

1. timelike geodesic if and only if $\left.h^{s}\right|_{\mathcal{V}} ^{\tilde{\mathcal{V}}_{\times \mathcal{V}}}=\left.h^{s}\right|_{\mathcal{H} \times \mathcal{H}} ^{\widetilde{\mathcal{H}}}=\left.h^{s}\right|_{\mathcal{V}} ^{\tilde{\mathcal{V}}_{\times \mathcal{H}}}=0$,

2. spacelike geodesic if and only if $\left.h^{s}\right|_{\mathcal{V} \times \mathcal{V}} ^{\widetilde{\mathcal{H}}}=\left.h^{s}\right|_{\mathcal{H} \times \mathcal{H}} ^{\widetilde{\mathcal{H}}}=\left.h^{s}\right|_{\mathcal{V} \times \mathcal{H}} ^{\widetilde{\mathcal{H}}}=0$,

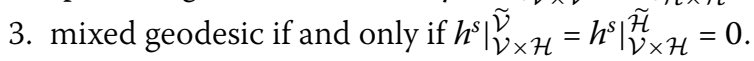

In view of Definition 3, we give the following proposition.

Proposition 3 Let $(M, g, S(T M))$ be an r-lightlike submanifold of a semi-Riemannian manifold $(\tilde{M}, \tilde{g})$ of index $(q+\tilde{q})$ and $S(T M)$ be an integrable distribution of index $q$. Then the following statements are true:

(a) The submanifold is timelike $\mathcal{V}$-geodesic and timelike $\mathcal{H}$-geodesic, then the mean curvature vector on $\Gamma(S(T M))$ is spacelike.

(b) The submanifold is spacelike $\mathcal{V}$-geodesic and spacelike $\mathcal{H}$-geodesic, then the mean curvature vector on $\Gamma(S(T M))$ is timelike.

Example 1 Let us consider the submanifold $M$ of the semi-Euclidean space $\mathbb{R}_{4}^{8}$ with the signature $(-,-,-,-,+,+,+,+)$ given by

$$
\phi\left(x_{1}, x_{2}, x_{3}, x_{4}\right)=\left(\frac{1}{\sqrt{2}} x_{1}, \cos x_{2}, \sin x_{2}, \sinh x_{3}, \cosh x_{3}, \frac{1}{\sqrt{2}} x_{1}, \frac{1}{\sqrt{2}} x_{4}, \frac{1}{\sqrt{2}} x_{4}\right)
$$

for all $\left(x_{1}, x_{2}, x_{3}, x_{4}\right) \in \mathbb{R}^{4}$. Then we have

$$
\begin{aligned}
& \xi_{1}=\frac{1}{\sqrt{2}} \frac{\partial}{\partial x_{1}}+\frac{1}{\sqrt{2}} \frac{\partial}{\partial x_{6}}, \quad e_{1}=-\sin x_{2} \frac{\partial}{\partial x_{2}}+\cos x_{2} \frac{\partial}{\partial x_{3}}, \\
& e_{2}=\cosh x_{3} \frac{\partial}{\partial x_{4}}+\sinh x_{3} \frac{\partial}{\partial x_{5}}, \quad e_{3}=\frac{1}{\sqrt{2}} \frac{\partial}{\partial x_{7}}+\frac{1}{\sqrt{2}} \frac{\partial}{\partial x_{8}}, \\
& N_{1}=-\frac{1}{\sqrt{2}} \frac{\partial}{\partial x_{1}}+\frac{1}{\sqrt{2}} \frac{\partial}{\partial x_{6}}, \quad e_{4}=\cos x_{2} \frac{\partial}{\partial x_{2}}+\sin x_{2} \frac{\partial}{\partial x_{3}}, \\
& e_{5}=\sinh x_{3} \frac{\partial}{\partial x_{4}}+\cosh x_{3} \frac{\partial}{\partial x_{5}}, \quad e_{6}=\frac{1}{\sqrt{2}} \frac{\partial}{\partial x_{7}}-\frac{1}{\sqrt{2}} \frac{\partial}{\partial x_{8}} .
\end{aligned}
$$

It is easy to see that $e_{1}, e_{2}, e_{4}$ are timelike unit vectors, $e_{3}, e_{5}, e_{6}$ are spacelike unit vectors and $M$ is a 1-lightlike submanifold with screen distribution $S(T M)=\operatorname{Span}\left\{e_{1}, e_{2}, e_{3}\right\}$, $\operatorname{Rad}(T M)=\operatorname{Span}\left\{\xi_{1}\right\}, \operatorname{ltr}(T M)=\operatorname{Span}\left\{N_{1}\right\}$, and $S\left(T M^{\perp}\right)=\operatorname{Span}\left\{e_{4}, e_{5}, e_{6}\right\}$.

Here, we have also

$$
S(T M)=\mathcal{V} \oplus_{\text {orth }} \mathcal{H}
$$


where $\mathcal{V}=\operatorname{Span}\left\{e_{1}, e_{2}\right\}$ and $\mathcal{H}=\operatorname{Span}\left\{e_{3}\right\}$ and

$$
S\left(T M^{\perp}\right)=\widetilde{\mathcal{V}} \oplus_{\text {orth }} \tilde{\mathcal{H}}
$$

where $\tilde{\mathcal{V}}=\operatorname{Span}\left\{e_{4}\right\}$ and $\tilde{\mathcal{H}}=\operatorname{Span}\left\{e_{5}, e_{6}\right\}$. By a straightforward computation, we have $B=$ $C=0$ and

$$
\begin{aligned}
& D_{11}^{4}=1, \quad D_{11}^{5}=D_{11}^{6}=0, \\
& D_{12}^{4}=D_{12}^{5}=D_{12}^{6}=D_{13}^{4}=D_{13}^{5}=D_{13}^{6}=0, \\
& D_{22}^{5}=1, \quad D_{22}^{4}=D_{22}^{6}=D_{23}^{4}=D_{23}^{5}=D_{23}^{6}=0, \\
& D_{33}^{4}=D_{33}^{5}=D_{33}^{6}=0 .
\end{aligned}
$$

Thus, we have

$$
\begin{array}{lll}
\left.|h|_{\mathcal{V} \times \mathcal{V}}^{\tilde{\mathcal{V}}}\right|^{2} \neq 0, & \left.|h|_{\mathcal{H} \times \mathcal{H}}^{\tilde{\mathcal{H}}}\right|^{2}=0, & \left.|h|_{\mathcal{V} \times \mathcal{H}}^{\tilde{\mathcal{H}}^{2}}\right|^{2}=0, \\
\left.|h|_{\mathcal{V} \times \mathcal{V}}^{\tilde{\mathcal{H}}}\right|^{2} \neq 0, & \left.|h|_{\mathcal{H} \times \mathcal{H}}^{\widetilde{\mathcal{H}}}\right|^{2}=0, & \left.|h|_{\mathcal{V} \times \mathcal{H}}^{\tilde{\mathcal{H}}}\right|^{2}=0,
\end{array}
$$

which shows that the submanifold is not timelike $\mathcal{V}$-geodesic and spacelike $\mathcal{V}$-geodesic but it is timelike $\mathcal{H}$-geodesic, spacelike $\mathcal{H}$-geodesic, and mixed geodesic.

Similarly, examples for the other cases can be given.

\section{Some relations for $r$-lightlike submanifolds}

We begin this section with the following definition.

Definition 4 Let $(M, g, S(T M))$ be an $(n+r)$-dimensional $r$-lightlike submanifold of a semi-Riemannian manifold and $S(T M)$ be an integrable distribution of index $q$. The bounded screen Ricci tensor, denoted by $\operatorname{Ric}_{S(T M)}$, is defined by

$$
\operatorname{Ric}_{S(T M)}(X, Y)=\operatorname{tr}\{Z \rightarrow R(X, Z) Y\}
$$

for any $X, Y \in \Gamma(S(T M))$.

Suppose $\left\{e_{1}, \ldots, e_{n}\right\}$ be an orthonormal basis of $\Gamma(S(T M))$. The bounded screen Ricci curvature at a unit vector $e_{i} \in \Gamma(S(T M))$, denoted by $\operatorname{Ric}_{S(T M)}\left(e_{i}\right)$, is given by

$$
\operatorname{Ric}_{S(T M)}\left(e_{i}\right)=\sum_{j \neq i=1}^{n} R\left(e_{i}, e_{j}, e_{j}, e_{i}\right)=\sum_{j \neq i=1}^{n} K_{i j} .
$$

We note that:

(a) If $n=1$, then the bounded screen Ricci curvature vanishes identically.

(b) If $n=2$, then the bounded screen Ricci curvature becomes the bounded sectional curvature.

Remark 1 We note that the screen Ricci curvature is bounded when the screen distribution of a lightlike submanifold is Riemannian. This map was first of all introduced by 
Duggal in [55] and named by the authors in $[56,57]$ in the case of a lightlike hypersurface of a Lorentzian manifold in which we know that $S(T M)$ is Riemannian.

Theorem 4 Let $(M, g, S(T M))$ be an $(r+3)$-dimensional $r$-lightlike submanifold of a semiRiemannian manifold and $S(T M)$ be an integrable distribution. The bounded screen Ricci curvature is constant at every unit vector on $\Gamma(S(T M))$ if and only if the bounded sectional curvature is constant.

Proof Let $\left\{e_{1}, e_{2}, e_{3}\right\}$ be an orthonormal basis of $\Gamma(S(T M))$. If $\operatorname{Ric}_{S(T M)}$ is constant, then we can write

$$
\begin{aligned}
& \operatorname{Ric}_{S(T M)}\left(e_{1}\right)=K_{12}+K_{13}=\lambda, \\
& \operatorname{Ric}_{S(T M)}\left(e_{2}\right)=K_{21}+K_{23}=\lambda, \\
& \operatorname{Ric}_{S(T M)}\left(e_{3}\right)=K_{31}+K_{32}=\lambda,
\end{aligned}
$$

where $\lambda$ is a constant. Thus, we have

$$
K_{12}=\frac{1}{2}\left[\operatorname{Ric}_{S(T M)}\left(e_{1}\right)+\operatorname{Ric}_{S(T M)}\left(e_{2}\right)-\operatorname{Ric}_{S(T M)}\left(e_{3}\right)\right]=\frac{1}{2} \lambda,
$$

which shows that $K_{12}$ is constant. The converse part of this theorem is straightforward.

Taking the trace in (18) with respect to $S(T M)$ and putting (35) in it, we have the following result.

Lemma 1 Let $(M, g, S(T M))$ be an $(n+r)$-dimensional $r$-lightlike submanifold of an $\tilde{m}$-dimensional semi-Riemannian manifold of index $(q+\tilde{q})$ and $S(T M)$ be an integrable distribution. Suppose $\left\{e_{1}, \ldots, e_{n}\right\}$ is an orthonormal basis of $\Gamma(S(T M))$. For any unit vector $X \in \Gamma(S(T M))$, we have

$$
\operatorname{Ric}_{S(T M)}(X)=\widetilde{\operatorname{Ric}}_{S(T M)}(X)+S(X)
$$

where

$$
\widetilde{\operatorname{Ric}}_{S(T M)}(X)=\varepsilon \sum_{j=1}^{n} \varepsilon_{j} \widetilde{R}\left(X, e_{j}, e_{j}, X\right), \quad g(X, X)=\varepsilon=\mp 1
$$

and

$$
\begin{aligned}
S(X)= & \varepsilon\left[\sum_{j=1}^{n} \varepsilon_{j}\left[\sum_{l=1}^{r} B^{l}\left(e_{j}, e_{j}\right) C^{l}(X, X)-\sum_{l=1}^{r} B^{\ell}\left(X, e_{j}\right) C^{\ell}\left(e_{j}, X\right)\right]\right. \\
& \left.-\sum_{j=1}^{n} \varepsilon_{j}\left[\sum_{\alpha=1}^{m} \varepsilon_{\alpha} D^{\alpha}\left(X, e_{j}\right) D^{\alpha}\left(e_{j}, X\right)-D^{\alpha}\left(e_{j}, e_{j}\right) D^{\alpha}(X, X)\right]\right] .
\end{aligned}
$$

Here, $\widetilde{\operatorname{Ric}}_{S(T M)}$ is the Ricci curvature of n-plane section (screen distribution) of $\widetilde{M}$ given in [21]. 
Theorem 5 Let $(M, g, S(T M))$ be an $(n+r)$-dimensional minimal $r$-lightlike submanifold of an $\tilde{m}$-dimensional semi-Riemannian space form $\widetilde{M}(c)$ and $S(T M)$ be an integrable distribution. For any spacelike unit vector $X \in \Gamma(S(T M))$, we have:

(a)

$$
\begin{aligned}
\operatorname{Ric}_{S(T M)}(X) \leq & (n-1) c+\left.\left.\left.\left|h^{\ell}\right|_{\mathcal{H}_{1} \times \mathcal{V}}|| h^{*}\right|_{\mathcal{H}_{1} \times \mathcal{V}}|-| h^{\ell}\right|_{\mathcal{H}_{1} \times \mathcal{H}}|| h^{*}\right|_{\mathcal{H}_{1} \times \mathcal{H}} \mid \\
& +\left.\left|h^{s}\right|_{\mathcal{H}_{1} \times \mathcal{V}}^{\widetilde{\mathcal{H}}}\right|^{2}+\left.\left|h^{s}\right|_{\mathcal{H}_{1} \times \mathcal{H}}\right|^{2}
\end{aligned}
$$

and

$$
\begin{aligned}
\operatorname{Ric}_{S(T M)}(X) \geq & (n-1) c+\left.\left.\left.\left|h^{\ell}\right|_{\mathcal{H}_{1} \times \mathcal{V}}|| h^{*}\right|_{\mathcal{H}_{1} \times \mathcal{V}}|-| h^{\ell}\right|_{\mathcal{H}_{1} \times \mathcal{H}}|| h^{*}\right|_{\mathcal{H}_{1} \times \mathcal{H}} \mid \\
& -\left.\left|h^{s}\right|_{\mathcal{H}_{1} \times \mathcal{V}}\right|^{2}-\left.\left|h^{s}\right|_{\mathcal{H}_{1} \times \mathcal{H}}^{\widetilde{\mathcal{H}}}\right|^{2},
\end{aligned}
$$

where $\mathcal{H}_{1}=\operatorname{Span}\{X\}$.

(b) The equality cases of both the inequalities (39) and (40) are true simultaneously for all spacelike vector $X \in \Gamma(S(T M))$ if and only if $D$ vanishes on $S(T M)$.

Proof (a) From (36) and (38) we get

$$
\begin{aligned}
\operatorname{Ric}_{S(T M)}\left(e_{i}\right)= & \sum_{j=1}^{n} \varepsilon_{i} \varepsilon_{j}\left[\sum_{l=1}^{r} B^{l}\left(e_{j}, e_{j}\right) C^{l}\left(e_{i}, e_{i}\right)-\sum_{l=1}^{r} B^{\ell}\left(e_{i}, e_{j}\right) C^{\ell}\left(e_{j}, e_{i}\right)\right. \\
& \left.+\sum_{\alpha=1}^{m} \varepsilon_{\alpha} D^{\alpha}\left(e_{j}, e_{j}\right) D^{\alpha}\left(e_{i}, e_{i}\right)-D^{\alpha}\left(e_{i}, e_{j}\right) D^{\alpha}\left(e_{j}, e_{i}\right)\right] \\
& +(n-1) c .
\end{aligned}
$$

Since $M$ is minimal we obtain

$$
\begin{aligned}
& \operatorname{Ric}_{S(T M)}(X)=(n-1) c+\left.\left|h^{\ell}{\mid \mathcal{H}_{1} \times \mathcal{V}}\right|\left|h^{*}\right|_{\mathcal{H}_{1} \times \mathcal{V}}|-| h^{\ell}{\mid \mathcal{H}_{1} \times \mathcal{H}}|| h^{*}\right|_{\mathcal{H}_{1} \times \mathcal{H}} \mid
\end{aligned}
$$

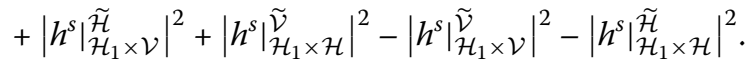

Taking into consideration (42), we have both the inequalities (39) and (40).

(b) The equality cases of both (39) and (40) inequalities are true simultaneously for all spacelike vector $X \in \Gamma(S(T M))$ if and only if

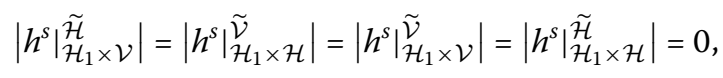

which implies that $D$ vanishes on $S(T M)$.

With similar arguments as in the proof of Theorem 5, we obtain the following theorem.

Theorem 6 Let $(M, g, S(T M))$ be an $(n+r)$-dimensional minimal $r$-lightlike submanifold of an $\tilde{m}$-dimensional semi-Riemannian space form $\widetilde{M}(c)$ and $S(T M)$ be an integrable distribution. For any timelike unit vector $Y \in \Gamma(S(T M))$, we have: 
(a)

$$
\begin{aligned}
\operatorname{Ric}_{S(T M)}(Y) \leq & (n-1) c-\left|h^{\ell}\right| \mathcal{V}_{1} \times \mathcal{V}|| h^{*}\left|\mathcal{V}_{1 \times \mathcal{V}}\right|+\left|h^{\ell}\right| \mathcal{V}_{1 \times \mathcal{H}}|| h^{*}\left|\mathcal{V}_{1 \times \mathcal{H}}\right| \\
& +\left.\left|h^{s}\right|_{\mathcal{V}_{1} \times \mathcal{V}}\right|^{2}+\left.\left|h^{s}\right|_{\mathcal{V}_{1} \times \mathcal{H}}\right|^{2}
\end{aligned}
$$

and

$$
\begin{aligned}
\operatorname{Ric}_{S(T M)}(Y) \geq & (n-1) c-\left|h^{\ell}\right| \mathcal{V}_{1} \times \mathcal{V}|| h^{*}\left|\mathcal{V}_{1} \times \mathcal{V}\right|+\left|h^{\ell}\right| \mathcal{V}_{1} \times \mathcal{H}|| h^{*}\left|\mathcal{V}_{1} \times \mathcal{H}\right| \\
& -\left.\left|h^{s}\right|_{\mathcal{V}_{1} \times \mathcal{V}}\right|^{2}-\left.\left|h^{s}\right|_{\mathcal{V}_{1} \times \mathcal{H}}\right|^{2},
\end{aligned}
$$

where $\mathcal{V}_{1}=\operatorname{Span}\{Y\}$.

(b) The equality cases of both the inequalities (44) and (45) are true simultaneously for all timelike vector $X \in \Gamma(S(T M))$ if and only if $D$ vanishes on $S(T M)$.

Now, we give the following definition.

Definition 5 Let $(M, g, S(T M))$ be an $(n+r)$-dimensional $r$-lightlike submanifold of semi-Riemannian manifold and $S(T M)$ be an integrable distribution of index $q$. Suppose $\left\{e_{1}, \ldots, e_{n}\right\}$ is an orthonormal basis of $\Gamma(S(T M))$. The bounded screen scalar curvature at a point $p \in M$, denoted by $r_{S(T M)}(p)$, is given by

$$
r_{S(T M)}(p)=\frac{1}{2} \sum_{i, j=1}^{n} K_{i j}
$$

With similar arguments to the proof of Theorem 4.7 in [56], we have the following proposition immediately.

Proposition 4 Let $(M, g, S(T M))$ be a $(2 n+r)$-dimensional r-lightlike submanifold and $S(T M)$ be an integrable distribution. Then the bounded screen Ricci curvature is constant if and only if

$$
r_{S(T M)}\left(\pi_{n}\right)=r_{S(T M)}\left(\pi_{n}^{\perp}\right)
$$

where $\pi_{n}$ is an $n$-dimensional non-degenerate sub-plane section of $\Gamma(S(T M))$ and $\pi_{n}^{\perp}$ is complementary vector bundle of $\pi_{n}$ in $\Gamma(S(T M))$.

Taking the trace in equation (36), we have the following result.

Lemma 2 Let $(M, g, S(T M))$ be an $(n+r)$-dimensional r-lightlike submanifold and $S(T M)$ be an integrable distribution. Then we have

$$
\begin{aligned}
2 r_{S(T M)}(p)= & 2 \tilde{r}_{S(T M)}(p)+n \mu_{1} \sum_{\ell=1}^{r}\left(\operatorname{trace} A_{N_{\ell}}\right)+n \mu_{2} \sum_{\alpha=1}^{m}\left(\operatorname{trace} A_{u_{\alpha}}\right) \\
& +2\left|h^{\ell}\right| \mathcal{V} \times \mathcal{H}|| h^{*}|\mathcal{V} \times \mathcal{H}|-\left|h^{\ell}\right| \mathcal{V} \times \mathcal{V}|| h^{*}|\mathcal{V} \times \mathcal{V}| \\
& -\left|h^{\ell}\right| \mathcal{H} \times \mathcal{H}|| h^{*}|\mathcal{H} \times \mathcal{H}|+\left|h^{s} \tilde{\mathcal{V}}_{\mathcal{V}}\right|^{2}+\left.\left|h^{s}\right|_{\mathcal{H} \times \mathcal{H}}\right|^{2} \\
& -\left.\left|h^{s}\right|_{\mathcal{\mathcal { H }} \times \mathcal{V}}^{\tilde{\mathcal{V}}}\right|^{2}-\left.\left|h^{s}\right|_{\mathcal{H} \times \mathcal{H}}^{\tilde{\mathcal{H}}}\right|^{2}+2\left|h^{s}\right| \mathcal{\mathcal { H }} \times\left.\mathcal{H}\right|^{2}-\left.2\left|h^{s}\right|_{\mathcal{V}}^{\tilde{\mathcal{V}} \times \mathcal{H}}\right|^{2},
\end{aligned}
$$


where

$$
\tilde{r}_{S(T M)}(p)=\frac{1}{2} \sum_{i, j=1}^{n} \widetilde{K}_{i j} .
$$

Here, $\tilde{r}_{S(T M)}\left(e_{i}\right)$ is the scalar curvature of $n$-plane section (screen distribution) of $\widetilde{M}$ given in [21].

Theorem 7 Let $(M, g, S(T M))$ be an $(n+r)$-dimensional $r$-lightlike submanifold of a semiRiemannian space form $\widetilde{M}(c)$ and $S(T M)$ be an integrable distribution. Then we have:

(a)

$$
\begin{aligned}
2 r_{S(T M)}(p) \leq & n(n-1) c+n \mu_{1} \sum_{\ell=1}^{r}\left(\operatorname{trace} A_{N_{\ell}}\right)+n \mu_{2} \sum_{\alpha=1}^{m}\left(\operatorname{trace} A_{u_{\alpha}}\right) \\
& +2\left|h^{\ell}\right| \mathcal{V} \times \mathcal{H}|| h^{*}|\mathcal{V} \times \mathcal{H}|-\left|h^{\ell}\right| \mathcal{V} \times \mathcal{V}|| h^{*}|\mathcal{V} \times \mathcal{V}| \\
& -\left|h^{\ell}\right|_{\mathcal{H} \times \mathcal{H}}|| h^{*}|\mathcal{H} \times \mathcal{H}|+\left.\left|h^{s}\right|_{\mathcal{V} \times \mathcal{V}}\right|^{2}+\left.\left|h^{s}\right|_{\mathcal{H} \times \mathcal{H}}\right|^{2} \\
& +\left.2\left|h^{s}\right|_{\mathcal{V} \times \mathcal{H}}^{\widetilde{\mathcal{H}}}\right|^{2} .
\end{aligned}
$$

The equality case of (50) is true for all $p \in M$ if and only if $M$ is spacelike $\mathcal{V}$-geodesic, spacelike $\mathcal{H}$-geodesic and timelike mixed geodesic.

(b)

$$
\begin{aligned}
2 r_{S(T M)}(p) \geq & n(n-1) c+n \mu_{1} \sum_{\ell=1}^{r}\left(\operatorname{trace} A_{N_{\ell}}\right)+n \mu_{2} \sum_{\alpha=1}^{m}\left(\operatorname{trace} A_{u_{\alpha}}\right) \\
& +2\left|h^{\ell}\right| \mathcal{V} \times \mathcal{H}|| h^{*}|\mathcal{V} \times \mathcal{H}|-\left|h^{\ell}\right| \mathcal{V} \times \mathcal{V}|| h^{*}|\mathcal{V} \times \mathcal{V}| \\
& -\left|h^{\ell}\right|_{\mathcal{H} \times \mathcal{H}}|| h^{*}|\mathcal{H} \times \mathcal{H}|-\left.\left|h^{s}\right|_{\mathcal{V} \times \mathcal{V}}^{\tilde{\mathcal{H}}}\right|^{2}-\left.\left|h^{s}\right|_{\mathcal{H} \times \mathcal{H}}^{\tilde{\mathcal{H}}}\right|^{2} \\
& -\left.2\left|h^{s}\right|_{\mathcal{V} \times \mathcal{H}}\right|^{2} .
\end{aligned}
$$

The equality case of (51) is true for all $p \in M$ if and only if $M$ is timelike $\mathcal{V}$-geodesic, timelike $\mathcal{H}$-geodesic and spacelike mixed geodesic.

Now, we recall a class of $r$-lightlike submanifolds of a semi-Riemannian manifold of an arbitrary signature which admits an integrable unique screen distribution as follows.

Definition 6 [47] An $r$-lightlike submanifold is called a screen locally conformal if

$$
C^{\ell}(X, Y)=\varphi_{\ell} B^{\ell}(X, Y), \quad \forall X, Y \in \Gamma\left(\left.T M\right|_{\mathcal{U}}\right), \ell \in\{1, \ldots, r\},
$$

where each $\varphi_{\ell}$ is a conformal smooth function on a neighborhood $\mathcal{U}$ in $M$. If each $\varphi_{\ell}$ is a non-zero constant, then the submanifold is called screen homothetic.

Lemma 3 [39] If $a_{1}, \ldots, a_{n}$ are $n$-real numbers $(n>1)$, then

$$
\frac{1}{n}\left(\sum_{i=1}^{n} a_{i}\right)^{2} \leq \sum_{i=1}^{n} a_{i}^{2},
$$

with equality if and only if $a_{1}=\cdots=a_{n}$. 
Theorem 8 Let $(M, g, S(T M))$ be an $(n+r)$-dimensional screen conformal $\left(\varphi_{\ell}>0\right)$ $r$-lightlike submanifold of an $\tilde{m}$-dimensional semi-Riemannian space form $\widetilde{M}(c), S(T M)$ be an integrable distribution of index $q$ and $S\left(T M^{\perp}\right)$ be Riemannian. Then we have

$$
\begin{aligned}
2 r_{S(T M)}(p) \leq & n(n-1) c+\sum_{\ell=1}^{r} n^{2} \varphi_{\ell} \mu_{1}^{2}+n \mu_{2} \sum_{\alpha=1}^{m}\left(\operatorname{trace} A_{u_{\alpha}}\right)-q \mu_{1}^{2} \mid \mathcal{V} \\
& -\left.(n-q) \mu_{1}^{2}\right|_{\mathcal{H}}+2 \varphi_{\ell}\left|h^{\ell}\right| \mathcal{V} \times\left.\mathcal{H}\right|^{2}+\left.2\left|h^{s}\right|_{\mathcal{V}}^{\widetilde{\mathcal{H}}}\right|^{2}
\end{aligned}
$$

The equality case of (54) is true for all $p \in M$ if and only if $h^{\ell}(X, X)=h^{\ell}(Y, Y)$ and $h^{s}(X, Y)=$ 0 for all two timelike or spacelike vectors $X, Y \in \Gamma(S(T M))$.

Proof Let $\left\{e_{1}, \ldots, e_{n}\right\}$ be an orthonormal basis of $\Gamma(S(T M))$. If $S\left(T M^{\perp}\right)$ is a Riemannian distribution, then we have $\tilde{\mathcal{V}}=0$. From equation (52), it follows that

$$
h^{*}(X, Y)=\varphi_{\ell} h^{\ell}(X, Y), \quad \forall X, Y \in \Gamma(T M) .
$$

Taking into account Lemma 3 and equation (55), we get

$$
\begin{aligned}
& \mu_{1} \sum_{\ell=1}^{n} \operatorname{trace} A_{N_{\ell}}=\sum_{\ell=1}^{n} n^{2} \varphi_{\ell} \mu_{1}^{2}, \\
& \left.q \mu_{1}^{2}|\mathcal{V} \leq| h^{\ell}\right|_{\mathcal{V} \times \mathcal{V}}|| h^{*}|\mathcal{V} \times \mathcal{V}|, \\
& \left.(n-q) \mu_{1}^{2}\right|_{\mathcal{H}} \leq\left.\left|h^{\ell}\right|_{\mathcal{H} \times \mathcal{H}}|| h^{*}\right|_{\mathcal{H} \times \mathcal{H}} \mid,
\end{aligned}
$$

where

$$
\mu_{1} \mid \mathcal{V}=-\frac{1}{q}\left(B\left(e_{1}, e_{1}\right)+\cdots+B\left(e_{q}, e_{q}\right)\right)
$$

and

$$
\left.\mu_{1}\right|_{\mathcal{H}}=\frac{1}{n-q}\left(B\left(e_{q+1}, e_{q+1}\right)+\cdots+B\left(e_{n}, e_{n}\right)\right) .
$$

If we put (56), (57), and (58) in (48), we obtain the inequality (54).

Assuming the equality case of (54), in view of Lemma 3 in (57) and (58), for each $\ell \in$ $\{1, \ldots, r\}$, we have

$$
B^{\ell}\left(e_{1}, e_{1}\right)=\cdots=B^{\ell}\left(e_{q}, e_{q}\right), B^{\ell}\left(e_{q+1}, e_{q+1}\right)=\cdots=B^{\ell}\left(e_{n}, e_{n}\right),
$$

and for each $i, j \in\{1, \ldots, q\}, a, b \in\{q+1, \ldots, n\}, \alpha \in\{1, \ldots, m\}$, we have

$$
D^{\ell}\left(e_{i}, e_{j}\right)=D^{\ell}\left(e_{a}, e_{b}\right)=0 .
$$

This completes the proof of the theorem. 
Authors' contributions

All authors contributed equally to the writing of this paper. All authors read and approved the final manuscript.

\section{Author details}

${ }^{1}$ Department of Mathematics, Faculty of Arts and Sciences, Inönü University, Malatya, 44280, Turkey. ${ }^{2}$ Department of Mathematics, Faculty of Arts and Sciences, Siirt University, Siirt, 56100, Turkey.

\section{Acknowledgements}

The authors are thankful to the referees for their valuable comments toward the improvement of the paper. The first author of this study is supported by $113 \mathrm{~F} 388$ coded project of the Scientific and Technological Research Council of Turkey (TÜBITAK)

Received: 28 October 2015 Accepted: 1 February 2016 Published online: 12 February 2016

\section{References}

1. Kulkarni, RS: The values of sectional curvature in indefinite metric. Comment. Math. Helv. 54, 173-176 (1979)

2. Nomizu, K: Remarks on sectional curvature of an indefinite metrics. Proc. Am. Math. Soc. 89(3), 473-476 (1983)

3. Dajczer, M, Nomuzi, K: On the boundedness of Ricci curvature of an indefinite metric. Bol. Soc. Bras. Mat. 11, 25-30 (1980)

4. Harris, SG: A triangle comparison theorem for Lorentz manifolds. Indiana Univ. Math. J. 31, 289-308 (1982)

5. Aktan, N, Sarikaya, MZ, Özüsağlam, E: B.Y. Chen's inequality for semi-slant submanifolds in T-space forms. Balk. J. Geom. Appl. 13(1), 1-10 (2008)

6. Alegre, P, Chen, B-Y, Munteanu, Ml: Riemannian submersions, $\delta$-invariants, and optimal inequality. Ann. Glob. Anal. Geom. 42(3), 317-331 (2012)

7. Arslan, K, Ezentas, R, Mihai, I, Murathan, C, Özgür, C: Certain inequalities for submanifolds in $(\kappa, \mu)$-contact space forms. Bull. Aust. Math. Soc. 64(2), 201-212 (2001)

8. Carriazo, A, Fernández, LM, Hans-Uber, MBBY: Chen's inequality for S-space forms: applications to slant immersions. Indian J. Pure Appl. Math. 34(9), 1287-1298 (2003)

9. Chen, B-Y: Some pinching and classification theorems for minimal submanifolds. Arch. Math. 60, 568-578 (1993)

10. Chen, B-Y: A Riemannian invariant and its application to submanifolds theory. Results Math. 27, 17-26 (1995)

11. Chen, B-Y: Mean curvature and shape operator of isometric immersions in real space forms. Glasg. Math. J. 38(1), 87-97 (1996)

12. Chen, B-Y: Pseudo-Riemannian Geometry, $\delta$-Invariants and Applications. World Scientific, Hackensack (2011)

13. Chen, B-Y, Dillen, F, Verstraelen, L: $\delta$-Invariants and their applications to centroaffine geometry. Differ. Geom. Appl. 22(3), 341-354 (2005)

14. Chen, B-Y, Dillen, F, Van der Veken, J, Vrancken, L: Curvature inequalities for Lagrangian submanifolds: the final solution. Differ. Geom. Appl. 31(6), 808-819 (2013)

15. Costache, S, Zamfir, I: An improved Chen-Ricci inequality for special slant submanifolds in Kenmotsu space forms. Ann. Pol. Math. 110, 81-89 (2014)

16. De Smet, PJ, Dillen, F, Verstraelen, L, Vrancken, L: A pointwise inequality in submanifold theory. Arch. Math. 35 115-128 (1999)

17. Decu, S, Haesen, S, Verstraelen, L: Optimal inequalities involving Casorati curvatures. Bull. Transilv. Univ. Brasov, Ser. B 14(49), 85-93 (2007)

18. Deng, S: Improved Chen-Ricci inequality for Lagrangian submanifolds in quaternion space forms. Int. Electron. J. Geom. 5(1), 163-170 (2012)

19. Dillen, F, Petrovic, M, Verstraelen, L: Einstein, conformally flat and semi-symmetric submanifolds satisfying Chen's equality. Isr. J. Math. 100(1), 163-169 (1997)

20. Gupta, RS, Ahmad, I, Haider, SMK: B.Y. Chen's inequality and its application to slant immersions into Kenmostu manifolds. Kyungpook Math. J. 44, 101-110 (2004)

21. Hong, S, Matsumoto, K, Tripathi, MM: Certain basic inequalities for submanifolds of locally conformal Kaehlerian space forms. SUT J. Math. 4(1), 75-94 (2005)

22. Kim, YH, Kim, DS: A basic inequality for submanifolds in Sasakian space forms. Houst. J. Math. 25, $247-257$ (1999)

23. Lee, CW, Yoon, DW, Lee, JW: Optimal inequalities for the Casorati curvatures of submanifolds of real space forms endowed with semi-symmetric metric connections. J. Inequal. Appl. 2014, Article ID 327 (2014)

24. Lee, JW, Vilcu, GE: Inequalities for generalized normalized $\delta$-Casorati curvatures of slant submanifolds in quaternionic space forms. Taiwan. J. Math. 19(3), 691-702 (2015)

25. Li, G, Wu, C: Slant immersions of complex space forms and Chen's inequality. Acta Math. Sci., Ser. B 25(2), 223-232 (2005)

26. Liu, X: On Ricci curvature of totally real submanifolds in a quaternion projective space. Arch. Math. 38, 297-305 (2002)

27. Matsumoto, K, Mihai, I, Tazawa, Y: Ricci tensor of slant submanifolds in complex space forms. Kodai Math. J. 26(1), 85-94 (2003)

28. Mihai, A: B.Y. Chen inequalities for slant submanifolds in generalized complex space forms. Rad. Mat. 12, 215-231 (2004)

29. Mihai, A, Özgür, C: Chen inequalities for submanifolds of real space forms with a semi-symmetric metric connection Taiwan J. Math. 14(4), 1465-1477 (2010)

30. Mihai, I, Al-Solamy, FR, Shahid, MH: On Ricci curvature of a quaternion CR-submanifold in a quaternion space form. Rad. Mat. 12(1), 91-98 (2003)

31. Oiagă, A, Mihai, I: B.-Y. Chen inequalities for slant submanifolds in complex space forms. Demonstr. Math. 32(4), 835-846 (1999)

32. Oprea, T: Chen's inequality in the Lagrangian case. Colloq. Math. 108, 163-169 (2007)

33. Özgür, C, Arslan, K: On some class of hypersurfaces in $E^{n+1}$ satisfying Chen's equality. Turk. J. Math. 26, 283-293 (2002)

34. Sasahara, T: On Chen invariant of CR-submanifolds in a complex hyperbolic space. Tsukuba J. Math. 26, 119-132 (2002) 
35. Suceavă, B: Some remarks on B.Y. Chen's inequality involving classical invariants. An. Ştiinţ. Univ. 'Al... Cuza' laşi, Mat. 45, 405-412 (1999)

36. Slesar, V, Şahin, B, Vilcu, GE: Inequalities for the Casorati curvatures of slant submanifolds in quaternionic space forms. J. Inequal. Appl. 2014, Article ID 123 (2014)

37. Shukla, SS, Rao, PK: Ricci curvature of quaternion slant submanifolds in quaternion space forms. Acta Math. Acad. Paedagog. Nyházi. 28(1), 69-81 (2012)

38. Tripathi, MM, Kim, JS, Kim, SB: A note on Chen's basic equality for submanifolds in a Sasakian space form. Int. J. Math. Math. Sci. 2003(11), 711-716 (2003)

39. Tripathi, MM: Certain basic inequalities for submanifolds in $(\kappa, \mu)$ space. In: Recent Advances in Riemannian and Lorentzian Geometries (Baltimore, MD, 2003). Contemp. Math., vol. 337, pp. 187-202. Am. Math. Soc., Providence (2003)

40. Uddin, S, Khan, KA: An inequality for contact CR-warped product submanifolds of nearly cosymplectic manifolds J. Inequal. Appl. 2012, Article ID 304 (2012)

41. Vilcu, GE: Inequalities for slant submanifolds in quaternionic space forms. Turk. J. Math. 34(1), 115-128 (2010)

42. Vilcu, GE: On Chen invariant and inequalities in quaternionic geometry. J. Inequal. Appl. 2013, Article ID 66 (2013)

43. Yoon, DW, Cho, KS: Inequality for warped products in generalized Sasakian space forms. Int. Math. J. 5(3), 225-235 (2004)

44. Zhang, P, Zhang, L, Song, W: Chen's inequalities for submanifolds of a Riemannian manifold of quasi-constant curvature with a semi-symmetric metric connection. Taiwan. J. Math. 18(6), 1841-1862 (2014)

45. Punzi, R, Schuller, FP, Wohlfarth, MNR: Geometric obstruction of black holes. Ann. Phys. 322, 1335-1372 (2007)

46. Schuller, FP, Wohlfarth, MNR: Sectional curvature bounds in gravity: regularisation of the Schwarzchild singularity. Nucl. Phys. B 698, 319-334 (2004)

47. Duggal, KL, Şahin, B: Differential Geometry of Lightlike Submanifolds. Birkhäuser, Basel (2010)

48. Duggal, KL, Jin, DH: Totally umbilical lightlike submanifolds. Kodai Math. J. 26, 49-68 (2003)

49. Bejan, CL, Duggal, KL: Global lightlike manifolds and harmonicity. Kodai Math. J. 28, 131-145 (2005)

50. Duggal, KL, Sahin, B: Generalized Cauchy-Riemann lightlike submanifolds. Acta Math. Hung. 112(1-2), 113-136 (2006)

51. Duggal, KL, Bejancu, A: Lightlike Submanifolds of Semi-Riemannian Manifolds and Applications. Mathematics and Its Applications, vol. 364. Kluwer Academic, Dordrecht (1996)

52. Griffiths, P, Harris, J: Principles of Algebraic Geometry. Wiley-Interscience, New York (1978)

53. Baum, H: Spin-Strukturen und Dirac-Operatoren über pseudoriemannschen Mannigfaltigkeiten (German) [English: Spin structures and Dirac operators on pseudo-Riemannian manifolds]. Teubner Texts in Math., vol. 41. Teubner, Leipzig (1981)

54. Nardmann, M: Pseudo-Riemannian metrics with prescribed scalar curvature. arXiv:math/0409435v2

55. Duggal, KL: On scalar curvature in lightlike geometry. J. Geom. Phys. 57(2), 473-481 (2007)

56. Gülbahar, M, KIIıc, E, Keleș, S: Chen-like inequalities on lightlike hypersurfaces of a Lorentzian manifold. J. Inequal. Appl. 2013, Article ID 266 (2013)

57. Gülbahar, M, KIlıç, E, Keleş, S: Some inequalities on screen homothetic lightlike hypersurfaces of a Lorentzian manifold. Taiwan. J. Math. 17, 2083-2100 (2013)

\section{Submit your manuscript to a SpringerOpen ${ }^{\ominus}$ journal and benefit from:}

- Convenient online submission

Rigorous peer review

Immediate publication on acceptance

- Open access: articles freely available online

- High visibility within the field

- Retaining the copyright to your article 